\title{
RAMAN SIGNATURE OF BONDING AND DISORDER IN CARBONS
}

\author{
A C FERRARI and J ROBERTSON, \\ Engineering Dept, Cambridge University, Cambridge CB2 1PZ, UK
}

\begin{abstract}
The factors controlling the position and intensity of the G and D peaks of the Raman spectra of disordered and amorphous carbons are separated in terms of a 3-stage model. The Raman spectra are shown to depend fundamentally on the degree of ordering of the $\mathrm{sp}^{2}$ sites, and only weakly or indirectly on the fraction of $\mathrm{sp}^{3}$ sites. Three factors control the $\mathrm{G}$ and D peaks; the perfection of graphitic order, replacing aromatic rings with olefinic chains and increasing the $\mathrm{sp}^{2}$ content. These rules allow us to state when the $\mathrm{G}$ peak position can be related reliably to $\mathrm{sp}^{3}$ content.
\end{abstract}

\section{INTRODUCTION}

Raman spectroscopy is widely used to characterise the microstructure of disordered graphite, amorphous carbon (a-C) and hydrogenated amorphous carbon $(\mathrm{a}-\mathrm{C}: \mathrm{H})[1-5]$. The bonding in the various types of a-C and $\mathrm{a}-\mathrm{C}: \mathrm{H}$ is defined in terms of their hydrogen content and fraction of $\mathrm{sp}^{3}$ bonding [6], as shown in Fig. 1. The key property of interest in a-C(:H) is the $\mathrm{sp}^{3}$ fraction. However, the usual methods to find $\mathrm{sp}^{3}$ content, NMR and electron energy loss spectroscopy (EELS), are time consuming so it would be very valuable to be able to use a rapid, non-destructive technique like Raman to derive the $\mathrm{sp}^{3}$ content.

\section{THEORY}

The Raman spectra of amorphous carbons for visible excitation are usually dominated by the features of graphitic carbon, the $\mathrm{G}$

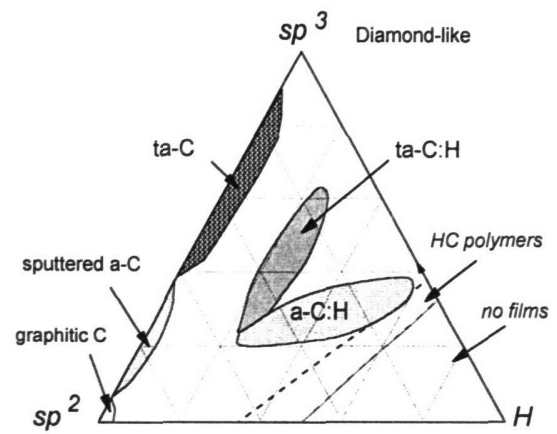

Fig. 1. Ternary phase diagram of $\mathrm{sp}^{3}$ and hydrogen contents of various forms of diamond-like carbon.

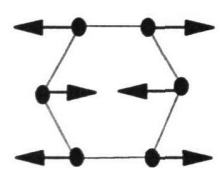

Fig. 2. G and D modes. peak around $1580 \mathrm{~cm}^{-1}$ and the $\mathrm{D}$ mode around $1350 \mathrm{~cm}^{-1}$. This is because visible Raman is $50-230$ times more sensitive to $\mathrm{sp}^{2}$ sites than sp ${ }^{3}$ sites, because visible photons preferentially excite their $\pi$ states, and even highly sp ${ }^{3}$ a-C still contains over $10 \% \mathrm{sp}^{2}$ sites. This means, as we show, that visible Raman is sensitive principally to the degree of order of the $\mathrm{sp}^{2}$ sites, and less sensitive to the fraction of $\mathrm{sp}^{3}$ bonding.

The bonding in disordered carbons consists of the $\sigma$ bonds of $\mathrm{sp}^{3}$ and $\mathrm{sp}^{2}$ sites and the $\pi$ bonds of $\mathrm{sp}^{2}$ sites [6]. The nature of $\sigma$ and $\pi$ bonds are different: $\sigma$ bonds are nearest-neighbor, 2-center, short-range bonds which fix the C-C skeleton of the 
lattice, while $\pi$ bonds are multi-center conjugated bonds giving rise to longer range forces. These longer range forces can favor $\mathrm{sp}^{2}$ sites arranging into graphitic clusters [6].

The $\mathrm{G}$ mode is a bond stretching vibration of a pair of $\mathrm{sp}^{2}$ sites, and occurs whether the $\mathrm{sp}^{2}$ sites are arranged as olefinic chains or aromatic rings (Fig. 2). The D mode is an $\mathrm{A}_{\mathrm{lg}}$ breathing vibration of a 6-fold aromatic rings, which is activated by

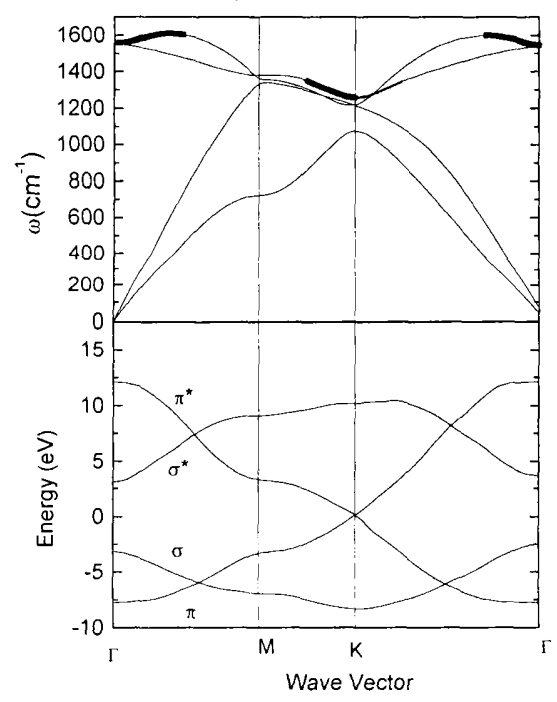

Fig. 3. Phonon dispersions and band structure of graphite sheet $[8,12]$. disorder [1]. It occurs only when $\mathrm{sp}^{2}$ sites are in aromatic rings.

Raman scattering is the inelastic scattering of a photon by a phonon due to the change in polarisability associated with that phonon mode. In a perfect crystal, the difference in energies of photons and phonons creates a $\mathrm{q}=0$ selection rule. For microcrystalline systems with grain size $\mathrm{L}$, the selection rule is relaxed to allow phonons of wavevector within $\Delta q=1 / L$ of the zone center $\Gamma$ to participate. For amorphous systems like a-Si, $\Delta \mathrm{q} \approx 1 /$ (bond length), and all phonons are allowed [7]. The Raman intensity is then the product of the Raman matrix element $C$, the vibration density of states $G$ and the Bose occupation factor $\mathrm{n}+1$,

$$
\mathrm{I}(\omega)=\frac{n(\omega)+1}{\omega} \cdot \mathrm{C}(\omega) \mathrm{G}(\omega)
$$

The visible Raman spectra of disordered carbon is different for 2 reasons. Firstly, visible photons of energy $2-2.5 \mathrm{eV}$ can only excite $\pi$ states, and for graphite they can only excite $\pi$ states over a relatively narrow part of the zone around the $K$ point $[9,10]$. Photons of energy $E$ resonantly excite electron states of wavevector $k$ whose $\pi-\pi^{*}$ band gap is $E(k)$, Fig. 3. This creates a polarisation wave of wavevector $k$. Secondly, $\pi$ states have a long-range polarisability so that this polarisation wave couples strongly to Raman-active breathing modes with a wavevector $\mathrm{q}=\mathrm{k}$ on the phonon dispersion curve (Fig. 3)[11,12]. This behaviour causes resonant enhancement of $\mathrm{sp}^{2}$ breathing modes such as the $\mathrm{D}$ modes, and means that the matrix element $\mathrm{C}(\omega)$ has a much stronger influence than the density of states (DOS) G( $\omega)$ on the visible Raman spectrum. This resonance and $\mathrm{q}=\mathrm{k}$ selection rule causes the $\mathrm{D}$ peak to disperse with changing photon energy [13].

Nanocrystalline graphite and a-C containing graphitic clusters behave in the same way because the electronic and vibrational modes of graphitic clusters can be folded onto a graphite lattice, as in a superlattice [11,12].

\section{THREE-STAGE MODEL}

We have found that the behaviour of Raman spectra in all types of microcrystalline and amorphous carbons can be classified using a 3-stage model [11]. The three stages of increasing amorphorisation are 


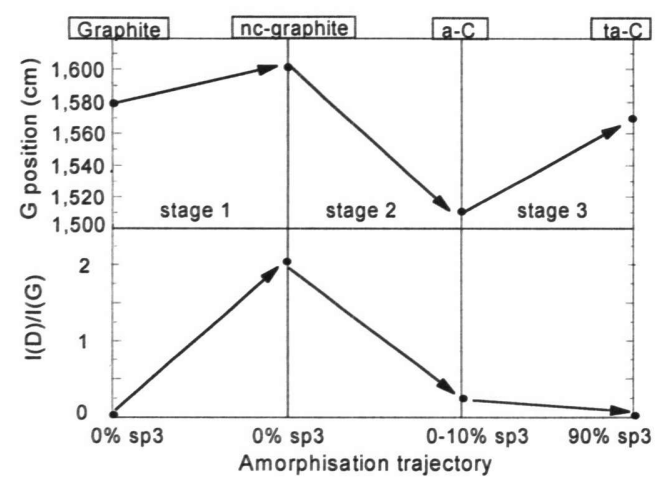

Fig. 4. Schematic variation of the $G$ position and $\mathrm{I}(\mathrm{D}) / \mathrm{I}(\mathrm{G})$ ratio during the 3 stages.
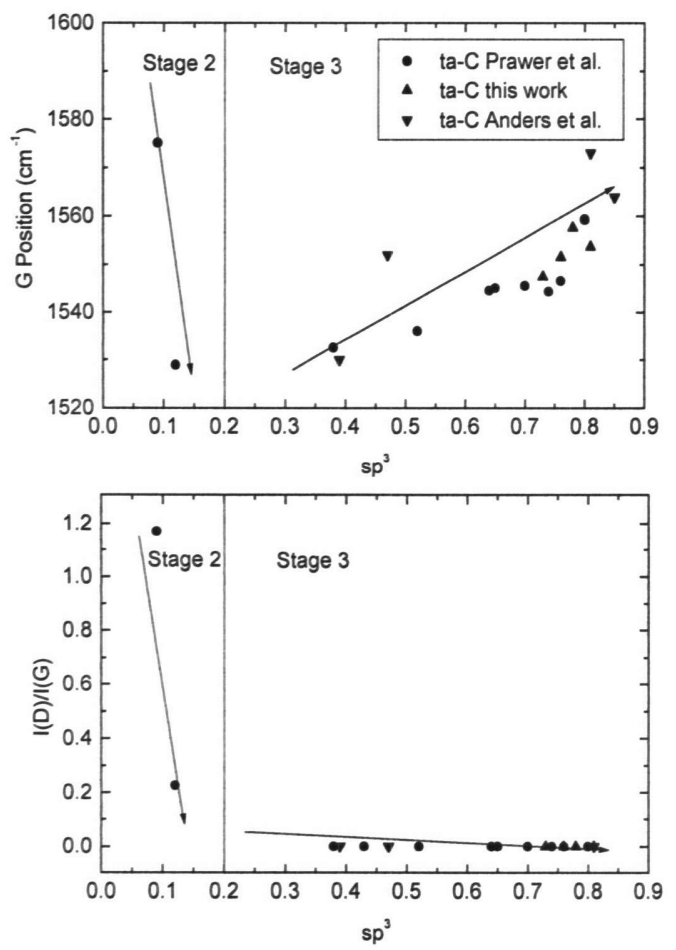

Fig. 5. Variation of $\mathrm{G}$ position and $\mathrm{I}(\mathrm{D}) / \mathrm{I}(\mathrm{G})$ for as-deposited ta-C [18-20]. When the cluster size falls below 1-2 nm, its internal disorder increases and the D intensity falls. The $\mathrm{G}$ peak maintains its intensity because it arises from all $\mathrm{sp}^{2}$ stretching modes. Thus, $I(D) / I(G)$ falls. We propose that $I(D) / I(G)$ varies with the number of ordered rings $M$, and so $I(D) / I(G)$ varies as
(1) graphite to nanocrystalline (nc-) graphite,

(2) nc-graphite to $\mathrm{sp}^{2} \mathrm{a}-\mathrm{C}$,

(3) $\mathrm{sp}^{2} \mathrm{a}-\mathrm{C}$ to $\mathrm{sp}^{3}$ ta-C.

Highly $\mathrm{sp}^{3}$ bonded a-C is refered to as tetrahedral amorphous carbon (ta-C). The G position and ratio of $D$ to $G$ peak intensities, $\mathrm{I}(\mathrm{D}) / \mathrm{I}(\mathrm{G})$, vary as shown schematically in Fig. 4.

Stage 1 corresponds to a loss of $\mathrm{q}$ selection within the VDOS of perfect graphite, due to a decrease of in-plane correlation length or grain size $\mathrm{L}_{\mathrm{a}}$. The main effects on the spectrum are; (a) a new sub-peak D' appears at 1600 $\mathrm{cm}^{-1}$, causing the $\mathrm{G}$ peak to shift upwards from $1580 \mathrm{~cm}^{-1}$ to 1600 $\mathrm{cm}^{-1}$; (b) the $\mathrm{D}$ peak intensity increases inversely with $\mathrm{L}_{\mathrm{a}}$ according to the well-known Tuinstra-Koenig relation [1],

$$
\mathrm{I}(\mathrm{D}) / \mathrm{I}(\mathrm{G})=\mathrm{B}(\lambda) / \mathrm{L}_{\mathrm{a}} \text {. }
$$

On the other hand, there is no dispersion of the $G$ position with $\lambda$, the laser wavelength.

Stage 2 corresponds to a loss of graphitic ordering, as nc-C is topologically disordered to give a-C by introducing 5,7,8-fold rings and other $\mathrm{sp}^{2}$ bonding configurations. The VDOS softens from that of graphite due to bond disorder. The end of stage [14]. The main effects on the Raman spectra are (a) the $G$ peak decreases from $1600 \mathrm{~cm}^{-1}$ to 1510 $\mathrm{cm}^{-1}$; (b) TK breaks down as I(D) decreases towards 0 ; (c) the G peak disperses. TK breaks down because the $\mathrm{D}$ peak is due to the correlated breathing of 6-fold rings. 2 corresponds to sputtered $\mathrm{sp}^{2} \mathrm{a}-\mathrm{C}$ 

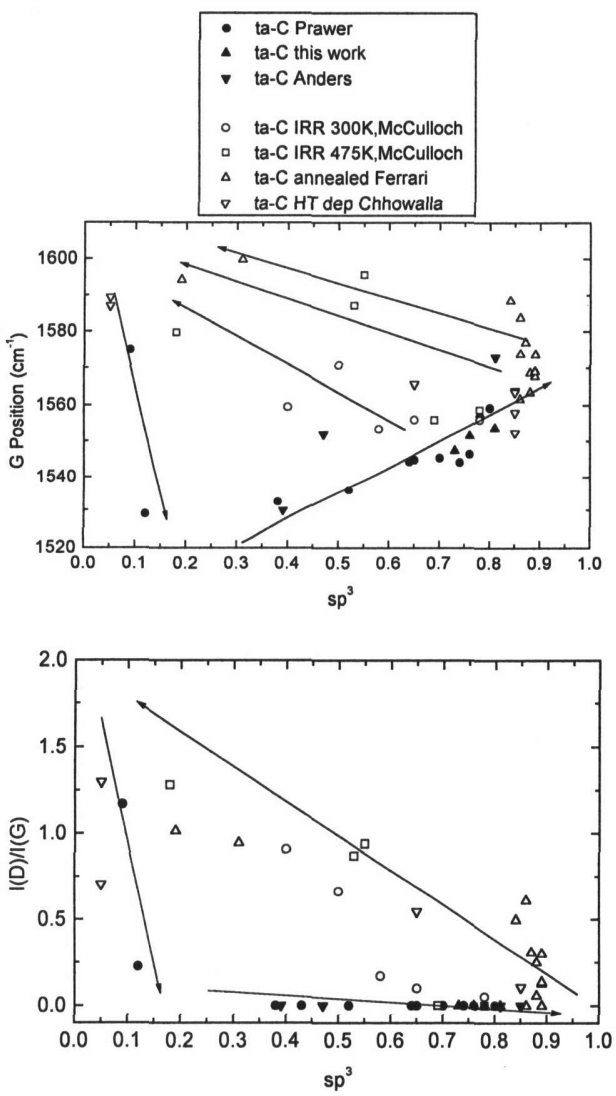

Fig. 6. G position and $\mathrm{I}(\mathrm{D}) / \mathrm{I}(\mathrm{G})$ ratio versus $\mathrm{sp}^{3}$ during annealing, showing hysteresis.

$$
\mathrm{I}(\mathrm{D}) / \mathrm{I}(\mathrm{G})=\mathrm{B}^{\prime} \cdot \mathrm{L}_{\mathrm{a}}{ }^{2} \text {. }
$$

A good example of stage 2 is the amorphisation of glassy carbon by irradiation [15]. Note that through the three stages, the development of the $\mathrm{D}$ peak indicates the disordering of graphite, but the ordering of a-C.

Stage 3 arises from the breaking up of the $\mathrm{sp}^{2}$ clusters as the $\mathrm{sp}^{3}$ content increases from $\sim 10 \%$ towards $100 \%$. The $\mathrm{sp}^{2}$ sites change first from rings to olefinic chains, and then to increasingly short chains $[16,17]$. $\mathrm{C}=\mathrm{C}$ chains have a shorter bond length than aromatic rings, so they have higher vibration frequencies of up to $1650 \mathrm{~cm}^{-1}$. The main effects on the Raman spectra are (a) the $G$ peak rises towards $1570 \mathrm{~cm}^{-1}$, and (b) $\mathrm{I}(\mathrm{D}) \approx$ 0 . A good example of stage 3 is as-deposited ta-C formed with a range of ion energies to vary its $\mathrm{sp}^{3}$ content $[18]$ (Fig. 5). Note that the high $\mathrm{G}$ position in ta-C is not due to high stress, as has been proposed [20]

The $\mathrm{G}$ peak is influenced by four factors in stages 2 and 3 ; disorder softens the VDOS and lowers $\mathrm{G}$, changing aromatic rings to olefinic chains raises $\mathrm{G}$, while mixing with $\mathrm{sp}^{3}$ modes tends to lower G. A unique behavior is possible if conditions lock the changes of $\mathrm{sp}^{2}$ ordering and $\mathrm{sp}^{3}$ fraction together. However, this is not always true. During for example thermal annealing of ta-C, existing $\mathrm{sp}^{2}$ sites begin to cluster and only at much higher temperatures do $\mathrm{sp}^{3}$ sites convert into more $\mathrm{sp}^{2}$ sites [19]. Such behavior causes a non-uniqueness or hysteresis in the dependence of Raman parameters on $\mathrm{sp}^{3}$ content, as shown in Fig. 6. This non-uniqueness restricts the situations where the $\mathrm{sp}^{3}$ fraction of a-C can be safely derived from visible Raman spectra.

\section{HYDROGENATED AMORPHOUS CARBON}

The $\mathrm{sp}^{3}$ fraction can be derived from visible Raman spectra for a-C:H deposited at room temperature by reactive sputtering or plasma enhanced chemical vapour deposition (PECVD). The main effect of hydrogen in the a-C:H network is to saturate $\mathrm{C}=\mathrm{C}$ bonds by converting them to $\mathrm{sp}^{3} \mathrm{CH}_{\mathrm{x}}$ groups. It does not particularly increase the fraction of $\mathrm{sp}^{3} \mathrm{C}-\mathrm{C}$ bonds. There are three bonding regimes in $\mathrm{a}-\mathrm{C}: \mathrm{H}$ as a function of 


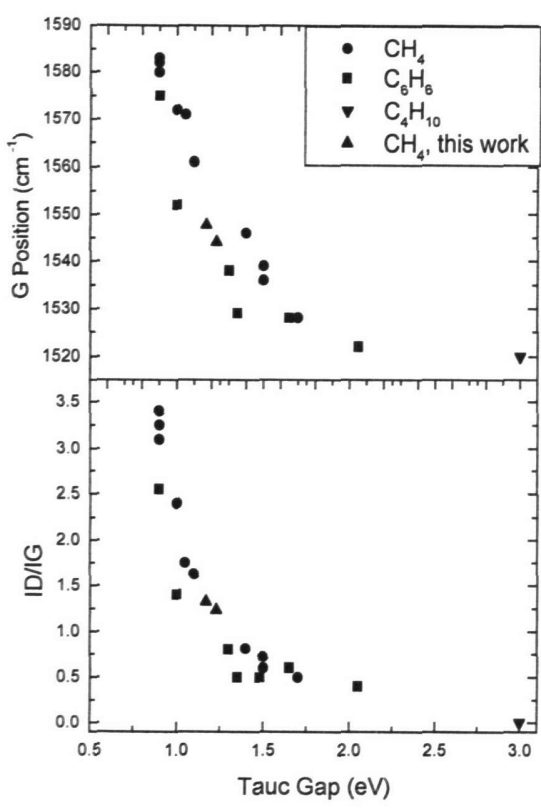

Fig. 7. G position and $I(D) / I(G)$ vs optical gap for a-C:H [5]

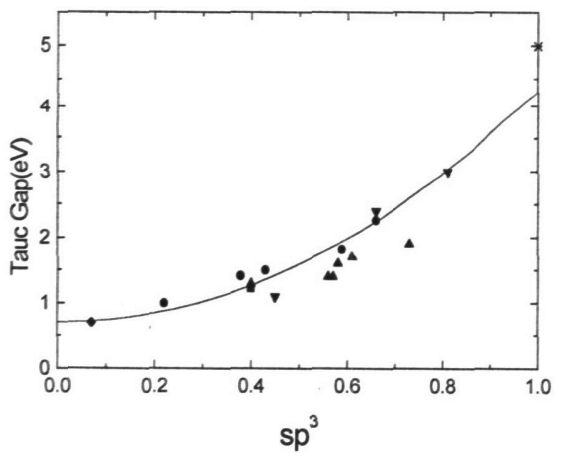

Fig. 9. Variation of optical gap with $\mathrm{sp}^{2}$ fraction, for a-C:H [23]. validated by NMR and EELS data where available [24], as shown in the Figure. Here, the $\mathrm{G}$ peak falls with increasing $\mathrm{sp}^{3}$ fraction. This is the opposite of what happens in ta-C. There is $\mathrm{G}$ peak dispersion, so the dependence on $\mathrm{sp}^{3}$ fraction becomes weaker for higher photon energies.

The hydrogenated analogue of ta- $\mathrm{C}$, ta- $\mathrm{C}: \mathrm{H}$ is made by deposition from high plasma density sources [25]. These have a higher fraction of $\mathrm{C}-\mathrm{C} \mathrm{sp}{ }^{3}$ bonding. Their 
$\mathrm{sp}^{2}$ order resembles that in ta- $\mathrm{C}$, with more short $\mathrm{C}=\mathrm{C}$ olefinic chains. This leads to a higher $\mathrm{G}$ position, for a given $\mathrm{sp}^{3}$ content compared to its position in a-C:H, as seen in Fig. 8 .

\section{REFERENCES}

1. F. Tuinstra and J.L. Koening, J. Chem. Phys. 53, 1126 (1970)

2. B. S. Elman, M. Shayegan, M. S. Dresselhaus, H. Mazurek and G. Dresselhaus, Phys. Rev. B, 25, 4142 (1982)

3. P. Lespade, R. Al-Jishi and M. S. Dresselhaus, Carbon, 20, 427 (1982)

4. J. Wagner, M. Ramsteiner, C. Wild, P. Koidl, Phys. Rev. B., 40, 1817 (1989)

5. M. A. Tamor and W. C. Vassel, J. Appl. Phys 76, 3823 (1994)

6. J. Robertson, Pure\&Appl. Chem., 66, 1789 (1994)

7. R. Alben, D. Weaire, J. E. Smith, M. H. Brodsky, Phys. Rev. B 11, 2271 (1975)

8. J. Robertson, Adv. Phys., 35, 317 (1986)

9. I. Pocsik, M. Hundhausen, M. Koos and L. Ley, J. Non-Cryst. Solids 227- 230, 1083 (1998)

10. M. J. Matthews, M. A. Pimenta, G. Dresselhaus, M. S. Dresselhaus and M. Endo, Phys. Rev. B, 59, 6585 (1999)

11. A C Ferrari, J Robertson, submitted to Phys Rev B (2000)

12. C. Mapelli, C. Castiglioni, G. Zerbi, K Mullen, Phys. Rev. B 6012710 (1999)

13. R. P. Vidano, D. B. Fishbach, L. J. Willis and T. M. Loehr, Solid State Comm. 39, $341(1981)$

14. F. Li, J. S. Lannin, Phys. Rev. Lett, 65, 1905 (1990);

15. D. G. McCulloch and S. Prawer, J. Appl. Phys. 78, 3040 (1995)

16. U. Stephan, T. Frauenheim, P. Blaudeck and J. Jungnickel, Phys. Rev. B, 49, 1489 (1994)

17. T. Kohler, T. Frauenheim and G. Jungnickel, Phys. Rev. B, 52, 11837 (1995)

18. S. Prawer, K. W. Nugent, Y. Lifshitz, G. D. Lempert, E. Grossman, J, Kulik, I. Avigal and R, Kalish, Diamond. Relat. Mater 5, 433 (1996)

19. A. C. Ferrari, B. Kleinsorge, N. A. Morrison, A. Hart, V. Stolojan and J. Robertson, J. Appl. Phys. 85, 7191 (1999)

20. S. Anders, J. W. Ager, G. M. Pharr, T. Y. Tsui and I. G. Brown, Thin Solid Films, 308, 186 (1997)

21. D. G. McCulloch, D. R. McKenzie, S. Prawer, A. R. Merchant, E. G. Gerstner and R. Kalish, Diamond. Relat. Mater., 6, 1622 (1997)

22. P Koidl, C Wagner, B Dischler, J Wagner, M Ramsteiner, Mat Sci Forum 5241 (1990)

23. J. Robertson, Phys Rev B 53, 16302 (1996)

24. M. A. Tamor, W. C. Vassel and K. R. Carduner, Appl. Phys. Lett. 58, 592 (1991)

25. M. Weiler, S. Sattel, T. Giessen, K. Jung, H. Ehrhardt, V. S. Veerasamy and J. Robertson, Phys. Rev. B, 53, 1594 (1996) 